\title{
Interactive Events Summary
}

\author{
H. Chad Lane ${ }^{1}$ and Brent Martin ${ }^{2}$ \\ AIED 2011 Interactive Events co-chairs \\ ${ }^{1}$ Institute for Creative Technologies \\ University of Southern California \\ Playa Vista, CA USA \\ lane @ict.usc.edu \\ ${ }^{2}$ Department of Computer Science and Software Engineering \\ University of Canterbury \\ Christchurch 8140, New Zealand \\ brent.martinecanterbury.ac.nz
}

The AIED 2011 organizing committee is pleased to present eleven interactive events at the $15^{\text {th }}$ International Conference on Artificial Intelligence in Education, held in Auckland, New Zealand. Interactive Events provide conference attendees a chance to experience many of the intelligent learning environments that the AIED community is building, from a learner's point of view. Attendees can ask questions of the researchers and students who have developed the systems and discuss new features and plans for the future. This year's program includes systems that support the learning sciences from all directions, including students, educators, and experimental researchers. Attendees can see work related to pedagogical agents, authoring systems, experimental tools, and educational games.

Three of the events involve the use of teachable agents, defined as systems that engage learners through learning-by-teaching. These include Betty's Brain (Segedy, et al.), DynaLearn (Beek, et al.), and Brick Game (Silvervarg, et al.). Three events will demonstrate the use of game-based approaches to learning including Monkey's Revenge (Rai, et. al.), Annie and FixIt (Thomas, et al.), and again, Brick Game. Two systems will demonstrate current approaches to pedagogical authoring, including ASTUS (Lebeau, et al.) and SimStudent (Matsuda, et al.). Two tutoring systems will be part of the Interactive Event program, including the constraint-based EER-Tutor for database systems (Weerashinghe, et al.) and Beetle II (Dzikovska, et al.), a dialogue system for reflection on circuit repair. Finally, Inquire for iPad (Spaulding, et al.), an interactive Biology textbook and DataShop (Stamper, et al.), a data repository and suite of analysis tools, will also be presented as interactive events.

In sum, the interactive events program at AIED 2011 is a highly international and thorough representation of contemporary research in the learning sciences. Full abstracts for all eleven AIED 2011 interactive events appear in these proceedings. 